\title{
A matter-dominated cosmological model with variable $G$ and $\Lambda$ and its confrontation with observational data
}

\author{
Ester Piedipalumbd* and Paolo Scudellaro \\ Dipartimento di Scienze Fisiche, \\ Università Federico II \\ and \\ Istituto Nazionale di Fisica Nucleare, \\ Sez. di Napoli, \\ Complesso Universitario di Monte S. Angelo, \\ Via Cintia, Ed. G, \\ I-80126 Napoli, Italy \\ Giampiero Esposito and Claudio Rubano \\ Istituto Nazionale di Fisica Nucleare, Sez. di Napoli, \\ Complesso Universitario di Monte S. Angelo, \\ Via Cintia, Ed. G, I-80126 Napoli, Italy
}

(Dated: February 22, 2018) 


\begin{abstract}
In the framework of renormalization-group improved cosmologies, we analyze both theoretically and observationally the exact and general solution of the matter-dominated cosmological equations, using the expression of $\Lambda=\Lambda(G)$ already determined by the integration method employed in a previous paper. A rough comparison between such a model and the concordance $\Lambda$ CDM model as to the magnitude-redshift relationship has been already done, without showing any appreciable differences. We here perform a more refined study of how astrophysical data (Union2 set) on type-I supernovae, gamma ray bursts (in a sample calibrated in a model independent way with the SneIa dataset), and gas fraction in galaxy clusters (using a sample of Chandra measurements of the X-ray gas mass fraction) affect the model and constrain its parameters. We also apply a cosmographic approach to our cosmological model and estimate the cosmographic parameters by fitting both the supernovae and the gamma ray bursts datasets. We show that this matter-dominated cosmological model with variable Newton parameter and variable cosmological term is indeed compatible with the observations above (on type Ia supernovae, the gamma ray bursts Hubble diagram, and the gas mass fraction in X-ray luminous galaxy clusters). The cosmographic approach adopted confirms such conclusions. Finally, it seems possible to include radiation into the model, since numerical integration of the equations derived by the presence of both radiation and matter shows that, after inflation, the total density parameter is initially dominated by the radiation contribution and later by the matter one.
\end{abstract}




\section{INTRODUCTION}

Pushed on by the overwhelming flow of observational data in the last fifteen years, most cosmologists today agree on a well defined cosmological paradigm, based on General Relativity plus a cosmological constant $\Lambda$. This paradigm is known as the Concordance Cosmological Model [1] and accounts not only for the early formation of large-scale structures but also for the more recently discovered stage of acceleration of the universe. As to the building up of galaxies and galaxy clusters, it has been necessary to introduce an ingredient like dark matter, which was first employed to succeed in describing rotational curves in spiral galaxies [2] (for an alternative view, see for example the work in Ref. [3]). On the other hand, cosmic acceleration requires the consideration of the so-called dark energy as the major ingredient of the cosmic content [4], $\Lambda$ being just the simplest way to consider it. Both of them sum up to more than $95 \%$ of the matter-energy constituents around us.

Such dark energies, on the other hand, have simply hidden the fundamental issues, since so far no exhaustive physical explanation for them has been put on firm theoretical and experimental ground. This has led to many alternative ways to reproduce the astrophysical phenomena cited above, not only by introducing theoretically well motivated new particles and fields, but also by means, for instance, of possible geometrical changes of the spacetime structure $(f(R)$ theories are well known examples of this kind of proposals [5] [6].

Without entering any details of so many attempts to describe the cosmological behaviour, we shall here consider only some aspects of one of them, i.e. that stemming from the possibility that not only the cosmological term $\Lambda$ could vary with space and time, but also the gravitational coupling $G$. In this context, one way to achieve the physical realization of such assumptions is to study cosmological dynamics by analyzing "renormalization group (RG) induced" quantum effects, which drive the dimensionless cosmological "constant" $\lambda(k)$ and Newton "constant" $g(k)$ from an ultraviolet attractive fixed point [7] [8] [9] [10] [11] [12]. In the exact theory, such a non-Gaussian ultraviolet fixed point implies its nonperturbative renormalizability [12] [13] [14] [15] [16] [17] [18] [19] [20]. As a result, this Renormalization Group -improved framework describes gravity at a typical distance scale $\ell \equiv k^{-1}$, and introduces an effective average action $\Gamma_{k}\left[g_{\mu \nu}\right]$ for Euclidean quantum gravity [8], finally implying an exact functional Renormalization Group equation for the $k$-dependence of $\Gamma_{k}$. This framework is usually known as quantum Einstein gravity. Within it, one can get 
an explicit $k$-dependence of both the running Newton and cosmological terms $G(k)$ and $\Lambda(k)$, which can be relevant both for the initial Planck era and the structure of black hole singularities [21] [22] [23].

Taking into account its inherent infrared divergences, quantum Einstein gravity can be subject to strong renormalization effects even at very large distances. In cosmology, such effects lead to a dynamical relaxation of $\Lambda$ and can also be assumed to deal with the cosmological constant problem [24]. Viewing the late accelerated expansion of the universe as a renormalization group evolution near a non-Gaussian infrared fixed point [25] (although the actual existence of an infrared fixed point has not been proved as yet), one can assume that the transition between standard FLRW cosmology and accelerated Renormalization Group driven expansion occurs at the time when the fixed point is almost reached. As to this, some agreement has been found between this kind of model and early SNeIa observations [26].

As a matter of fact, for a homogeneous and isotropic universe, it is possible to identify $k$ with the inverse of cosmological time, $k \propto 1 / t$ [21] [25], hence deriving a dynamical evolution for $G(k)$ and $\Lambda(k)$ induced by their Renormalization Group running. The Arnowitt-DeserMisner (ADM) formulation [27] builds a modified action functional which reduces to the Einstein-Hilbert action when $G$ is constant. Within such a framework, and always assuming homogeneity and isotropy, one can obtain a power-law growth of the scale factor for pure gravity and for a massless $\varphi^{4}$ theory, in agreement with what is known on fixed-point cosmology. On the other hand, by means of the so-called Noether Symmetry Approach [28] [29], in Ref. [30] we have also proposed solutions for the pure gravity case, which mimic inflation without introducing a scalar field in the cosmic content. In Ref. [31], for gravity with a scalar field, this approach only succeeds in fixing the expressions of $\Lambda(G)$ and $V(\varphi)$ as $\Lambda \propto G$ and $V \propto \varphi^{2}$, respectively, while the transformed cosmological equations derived by means of the method do not seem to be easily solvable [31], therefore giving no new insight into possible solutions.

In what follows, we take again into account the exact solutions of the flat dust matterdominated cosmological equations (without any scalar field), already investigated in Ref. [32] by means of the Noether Symmetry Approach, and by using an expression for $\Lambda=\Lambda(G)$ determined by the method itself. After briefly reviewing the theoretical model, we show that our cosmological model is compatible with various recent observational data, in particular with the observations of type Ia supernovae (SNeIa) (we use the recently updated SNeIa 
sample, referred to as Union2 [33], containing 557 SNeIa spanning the redshift range $0.015 \leq$ $z \leq 1.55$.), the Gamma Ray Bursts Hubble diagram (GRBs HD) (we use a sample calibrated in a model independent way with the SneIa dataset [34]), and the gas mass fraction in Xray luminous galaxy clusters (we use a sample of Chandra measurements of the X-ray gas mass fraction in 42 hot $(k T>5 \mathrm{keV})$, X-ray luminous, dynamically relaxed galaxy clusters spanning the redshift range $0.05<z<1.1[35]$ ).

We are aware that such observational tests cannot definitively distinguish our model from the standard $\Lambda \mathrm{CDM}$ one, at least because of the presence of still large observational errors. However, it provides a crucial and necessary test of reliability.

Looking at future studies on this, we finally apply to our cosmological model a cosmographic approach, which can indeed contribute to select realistic models without imposing arbitrary choices a priori. As a matter of fact, cosmography and its reliability are based on the assumptions that the universe is spatially homogeneous and isotropic on large scale, and luminosity distance can be "tracked" by the derivative series of the scale factor $a(t)$. We actually estimate the cosmographic parameters here derived by fitting both the SNeIa Union2 dataset and the calibrated GRBs HD.

We also begin to study how our model is affected by the inclusion of radiation into the cosmic content. It in fact turns out that, by performing the numerical integration of the equations so rewritten, the total density parameter is initially dominated by the radiation contribution and later on by the matter one, leaving then space to the now observed accelerated stage.

The scheme of the paper is as follows. In Section 2 we summarize the Lagrangian formulation used to derive the Renormalization-Group improved Einstein cosmological equations with the ordinary matter energy-momentum tensor, as well as the results deduced from the Noether symmetry found in Ref. [32]. In Sections 3, 4 and 5 we present the comparison of theoretical predictions with observational data. Section 6 is then devoted to the cosmographic approach, and Section 7 to inclusion of radiation into the model. Finally, some conclusions are drawn in Section 8. 


\section{THEORETICAL MODEL}

Let us consider the approach outlined in Ref. [27] [30] [32] [31] and there applied to models of gravity with variable $G$ and $\Lambda$ in the context of quantum Einstein gravity. It is known that, in a homogeneous and isotropic universe, an independent dynamical $G$ is equivalent to metric-scalar gravity already at classical level [36] [37], while independent variations (with position and time) of $G$ and $\Lambda$ can lead to pathological situations. Indeed, if $\Lambda$ were an independent variable, one should write that the momentum conjugate to it vanishes, and the preservation in time of this primary constraint would imply a vanishing lapse function and hence a "collapse" of spacetime geometry [27]. All this in fact leads to assume a generic functional dependence $\Lambda=\Lambda(G)$ [27].

In the matter-dominated case in a flat homogeneous and isotropic cosmology (with a signature,,,-+++ for the metric, lapse function $N=1$ and shift vector $N^{i}=0$ ), as in Ref. [32], we start from the Lagrangian

$$
L=\frac{1}{8 \pi G}\left(-3 a \dot{a}^{2}-a^{3} \Lambda+\frac{1}{2} \mu a^{3} \frac{\dot{G}^{2}}{G^{2}}\right)-D a^{-3(\gamma-1)},
$$

where $G=G(t)$ and $\Lambda=\Lambda(G(t))$, while dots indicate time derivatives, and $\mu$ is a nonvanishing interaction parameter introduced in Ref. [27] and also used in Ref. [32], where it was shown that $\mu>2$. The matter contribution is of course given by $L_{m} \equiv-D a^{-3(\gamma-1)}$, with $1 \leq \gamma \leq 2$; here, we have to take $\gamma=1$ for dust, while $D$ is a suitable integration

constant connected to the matter content. As shown in Ref. [32], from Eq. (11) we get the Euler-Lagrange equations for $a$ and $G$

$$
\begin{gathered}
\frac{\ddot{a}}{a}+\frac{\dot{a}^{2}}{2 a^{2}}-\frac{\Lambda}{2}-\frac{\dot{a} \dot{G}}{a G}+\frac{\mu \dot{G}^{2}}{4 G^{2}}=0 \\
\mu \ddot{G}-\frac{3}{2} \mu \frac{\dot{G}^{2}}{G}+3 \mu \frac{\dot{a}}{a} \dot{G}+\frac{G}{2}\left(-6 \frac{\dot{a}^{2}}{a^{2}}-2 \Lambda+2 G \frac{d \Lambda}{d G}\right)=0 .
\end{gathered}
$$

The Hamiltonian constraint [32]

$$
\frac{\dot{a}^{2}}{a^{2}}-\frac{\Lambda}{3}-\frac{\mu}{6} \frac{\dot{G}^{2}}{G^{2}}-\frac{8 \pi G}{3} D a^{-3}=0
$$

is equivalent to the constraint on the energy function associated with $L$ [28] [29] [30] [32] [31]

$$
E_{L} \equiv \frac{\partial L}{\partial \dot{a}} \dot{a}+\frac{\partial L}{\partial \dot{G}} \dot{G}-L=0
$$


For matter, the dust case involves a zero pressure, $p_{m}=0$, and an energy density $\rho_{m}=$ $D a^{-3}$, so that the matter term in the Lagrangian is simply a constant. It therefore has no effect on the equations of motion with respect to the pure gravity case; nevertheless, it has to be considered, since it occurs in the constraint equation (5). The system of equations of motion can then be solved [32] by using the Noether Symmetry Approach [28] [29], in which we consider $L$ as a point Lagrangian, a function of the variables $a$ and $G$, and their first derivatives [28] [29] [30] [32]. We have already shown that a consistent choice of the function $\Lambda=\Lambda(G)$ leads to the existence of a Noether symmetry for the Lagrangian [32]. As a matter of fact, in the matter-dominated case we get the same Noether symmetry as in the pure gravity situation, so that, by using the same transformations introduced in this latter case, one can write $a=a(t)$ and $G=G(t)$ as therein, now just updating the energy constraint. (For more details, see Ref. [32].)

The dynamics of $\Lambda$ is coupled with that of $G$ and is driven by the equation

$$
2(1-J) \Lambda+G \frac{d \Lambda}{d G}=0,
$$

where the parameter $J$ is an arbitrary constant related to the interaction factor $\mu$ by the relation $\mu=\frac{2}{3}(3-2 J)^{2} \neq 0, \frac{2}{3}$. This equation admits the solution

$$
\Lambda=\Lambda(t ; n)=W G^{\frac{1}{1-3 n}},
$$

$W$ being an integration constant and $n(J) \equiv \frac{3-2 J}{6(1-J)}$. It turns out that $W$ is related to the present value of $G$ [32], and we might determine $W$ in order to get $G_{0} \equiv G_{N} \equiv G_{\text {Newton }}$.

Furthermore, we fix time scale and origin so as to get $a(0)=0$. Thus, we find [32]

$$
\begin{aligned}
a & =a(t)=A\left(t^{\frac{1}{6 n-1}+1}\left(B+t^{\frac{1}{6 n-1}+1}\right)\right)^{n}, \\
G=G(t) & =C\left(t^{2}+B t^{\frac{2-6 n}{1-6 n}}\right)^{3 n-1},
\end{aligned}
$$

where we define the constants

$$
\begin{aligned}
& A \equiv A(n, W) \equiv 12^{\frac{n(1+6 n)}{1-6 n}} n^{\frac{12 n^{2}}{1-6 n}}(6 n-1)^{\frac{12 n^{2}}{6 n-1}}(12 n-1)^{-n} W^{n}, \\
& B \equiv B(n, W, D) \equiv W^{-1}\left[2^{\frac{3(1-10 n)}{1-6 n}}(3 n)^{\frac{6 n}{6 n-1}}(6 n-1)^{\frac{1-12 n}{6 n-1}}(12 n-1) \pi D\right], \\
& C \equiv C(n, W) \equiv(6 n-1)^{2(3 n-1)}\left[12 n^{2}(12 n-1)\right]^{1-3 n} W^{3 n-1} .
\end{aligned}
$$

Here we point out that the asymptotic time behavior of the scale factor is characterized by the two exponents

$$
p_{1} \equiv \frac{12 n^{2}}{(6 n-1)}, p_{2} \equiv \frac{6 n^{2}}{(6 n-1)},
$$


and we want that $p_{1}>1$ and $p_{2}<1$ (in order to obtain early matter domination and a later accelerated evolution ), which implies that we find a limited range of variability for the $n$ parameter[56], $(3-\sqrt{3}) / 6<n<(3+\sqrt{3}) / 6$. We then see that, when $D=0$, one has $B=0$, and we recover the same results obtained in Ref. [31] for the pure gravity model.

Eqs. (10) and (11) make it possible to obtain $D$ and $W$ as functions of $A, B$ and $n$, i.e.

$$
\begin{aligned}
D & \equiv \frac{2^{\frac{2}{6 n-1}-3} 3^{\frac{1}{6 n-1}} n^{\frac{1}{6 n-1}+1}(6 n-1)^{\frac{1}{1-6 n}} A^{\frac{1}{n}} B}{\pi}, \\
W & \equiv 1^{\frac{2}{6 n-1}+1} n^{\frac{12 n}{6 n-1}}(6 n-1)^{\frac{12 n}{1-6 n}}(12 n-1) A^{\frac{1}{n}} .
\end{aligned}
$$

To make things analytically simpler and obtain a better control of the space of parameters related to the integration constants, we set the present time $t_{0}=1$. This fixes the scale of time according to the (unknown) age of the universe. In other words, this means that we are using the age of the universe, $t_{0}$, as a unit of time, and the whole history of the universe has been squeezed to the range of time $[0,1]$. We then set $a_{0}=a(1)=1$, which is standard, and finally $H_{0}=H(1) \simeq 1$. Because of our choice of time unit, it turns out that our $H_{0}$ is not the same as the Hubble constant which appears in the standard FLRW model.

Such choices introduce a constraint between $A$ and $B$ :

$$
A=(1+B)^{-n}
$$

On choosing to normalize $a_{0}$, the definition of the redshift $z \equiv a_{0} / a-1=1 / a-1$ yields

$$
z=z(t)=(B+1)^{n} t^{\frac{6 n^{2}}{1-6 n}}\left(B+t^{\frac{1}{6 n-1}+1}\right)^{-n}-1
$$

depending only on the parameter $n$. It turns out that we obtain the following expression for $H_{0}$ and $\Lambda_{0}$ :

$$
\begin{gathered}
H_{0} \equiv H\left(t_{0}=1\right)=\frac{6(2+B) n^{2}}{(1+B)(-1+6 n)}, \\
\Lambda_{0} \equiv \Lambda\left(t_{0}=1\right)=-\frac{6(B+2) n^{2}(B+1)^{n-1}}{6 n-1} .
\end{gathered}
$$

We have constrained the parameters of our model in order to have $G_{0}=G_{N}=1$. Therefore, the density parameter of matter is

$$
\left.\Omega_{m} \equiv \frac{8 \pi G(t, B, n) D}{3 H(t, B, n)^{2}}\right|_{t=1} \equiv \frac{8 \pi G_{0} D}{3 H_{0}^{2}}=\frac{B(B+1)(6 n-1)}{9(B+2)^{2} n^{2}}
$$


and it turns out that the constraint

$$
\Omega_{m}+\Omega_{\Lambda 0}+\Omega_{G 0}=1
$$

is satisfied. Moreover, as already pointed out in Ref. [38], we do not expect that $G_{0} \equiv G_{N} \equiv$ $G_{\text {Newton}}$, even if we fixed the parameters in order to get it. Anyway, we have to consider that small differences between $G_{0}$ and $G_{N}$ could imply $G_{0}$ varying a lot in time, with relevant effects on the evolution of the universe. It is worth noting that $G$ has a special role in the subject of time variation of the fundamental parameters. Actually a dependence of $G$ on time may point out to violation of the strong equivalence principle, but not necessarily of the Einstein equivalence principle, whereas the nonconstancy of the other "constants", like the electroweak or strong coupling constants, necessarily represents a violation of the equivalence principle in both its forms. Here we will only discuss some aspects of the response of primordial abundances to the time variations of $G$, as expected in our model, on the basis of previous analysis on the subject performed in Ref. [39].

It turns out that the production of each element responds in its own way to a variation $\delta G$ of the Newton constant. A general study, that can account for a time dependence of $G$ during the BBN period, requires the introduction of suitable functions which describe the response of each element to an arbitrary time-dependent modification of the early universe expansion rate, and it is out of the aim of the present work. So, we simply discuss the observational bounds on the possible variations of the gravitational constant in the early universe, considering the best limit (at $3 \sigma), \delta G=0.09_{-0.19}^{+0.22}$, obtained in Ref. [39], by combining ${ }^{2} \mathrm{H}$ observational results with the measurements of the baryon to photon ratio obtained from CMB and LSS data[57]. We will postpone to a forthcoming paper the detailed analysis of the dependence of the various elemental abundances on the time variation of $G$ for our model. It turns out that our model can satisfy such a best fit limit, provided that the $n$ parameter is appropriately selected (the role of $B$ is only marginal with respect to this strong constraint), and compatible with the other basic cosmological observations, as shown in Fig. 1,

To further investigate this issue, we first evaluate the fractional time rate of change of $G$

$$
\frac{\dot{G}}{G_{0}} \sim \times 10^{-2} .
$$

Here, it is important to remember that we are using the age of the universe as unit, so that 


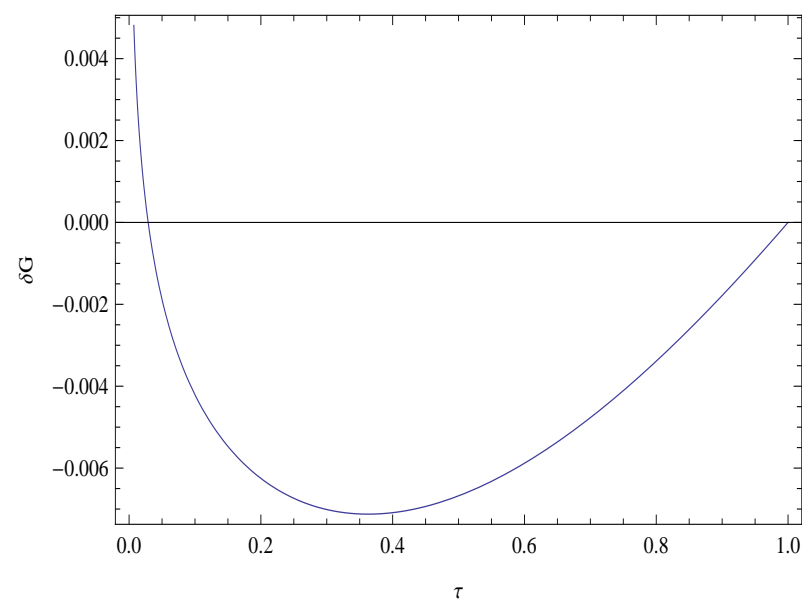

FIG. 1: Time evolution for the relative variation $\delta G$ for our model, with $B=2.77, n=0.32$. As we will discuss in the next sections such values are fully consistent with the constraints resulting from other cosmological datasets.

the effective rate is of order $10^{-13} \mathrm{yr}^{-1}$, in agreement with the observations (see for instance Ref. [40]).

We can thus say that, eventually, the running of both the gravitational coupling $G=G(t)$ and the cosmological term $\Lambda=\Lambda(t)$ induced by quantum effects appears to yield both a primordial inflation (soon after the universe exits the region where the attraction basin of the non-Gaussian (ultraviolet) fixed point works [38]), and a later inflationary epoch in a matter-dominated period of the expansion of the universe. This appears to be interesting since it is obtained always without having to introduce any scalar field in the cosmic content [32].

\section{Re-parametrization of the model}

Let us now exhibit a re-parametrization of our model in terms of $H_{0}$ and $\Omega_{m}$ instead of $B$ and $n$. On inverting the systems of Eqs. (18) and (20) it is indeed possible to recover the parameters $B$ and $n$ as functions of $H_{0}$ and $\Omega_{m}$; actually, we have that

$$
\begin{aligned}
B & =\frac{6 H_{0} \Omega_{m}}{2-3 H_{0} \Omega_{m}}, \\
n & =\frac{1}{24}\left(\sqrt{3} \sqrt{H_{0}\left(3 H_{0} \Omega_{m}+2\right)\left(9 H_{0}^{2} \Omega_{m}+6 H_{0}-8\right)}+9 H_{0}^{2} \Omega_{m}+6 H_{0}\right) .
\end{aligned}
$$

The conditions on the two exponents $p_{1}$ and $p_{2}$ (that is $p_{1}>1$ and $p_{2}<1$ ) give rise to a 


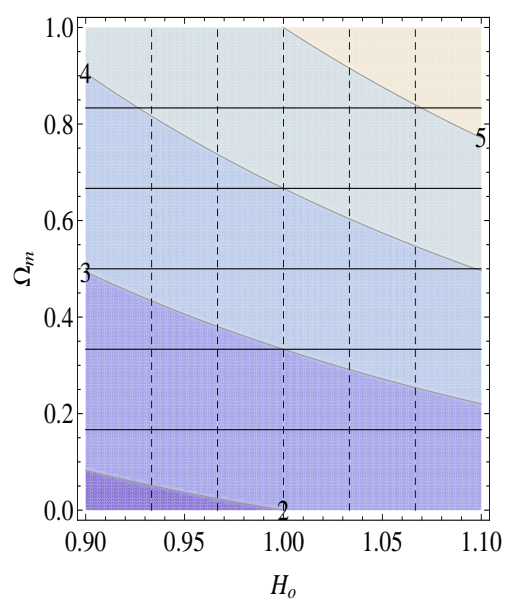

FIG. 2: Allowed regions (in light blue) of the space of parameters $n$ and $B$.

constraint on the space of parameters for $H_{0}$ and $\Omega_{m}$, as shown in Fig. 2; actually, it turns out that

$$
2<H_{0}\left(3 H_{0} \Omega_{m}+2\right)<4
$$

It is worth noting that the space of parameters $H_{0}$ and $\Omega_{m}$ is reasonably deducible from physical arguments. As a matter of fact, $\Omega_{m}$ varies in the range $[0,1]$, and the range of variation for $H_{0}$ can be inferred by assuming that the age of the universe can be written in the following way:

$$
t_{0}=\gamma \times 1 G y=3.15 \cdot 10^{16} \gamma s
$$

where $\gamma$ is a constant to be determined by astronomical observations. With this definition the value of $H_{0}$ can be related to the small $h=\bar{H}_{0} / 100$ of the standard FLRW model. It turns out that

$$
H_{0}=0.1 h \gamma \text {. }
$$

If we accept that $t_{0}=13.76 \pm 0.11 G y$ and $h=0.71 \pm 0.014$, as given by WMAP7 [41], then the region of variability at $2 \sigma$ for $H_{0}$ turns out to be $(0.92,1.03)$. It is interesting to note that the constraint in Eq. (25) is satisfied in the whole domain of $H_{0}$ and for $0.06<\Omega_{m}<0.6$.

\section{CONSTRAINTS FROM RECENT SNEIA OBSERVATIONS}

Over the last years the confidence in type Ia supernovae as standard candles has been steadily growing. Actually, it was just the SNeIa observations that gave the first strong 
indication of an accelerating expansion of the universe, which can be accounted for by assuming the existence of some kind of dark energy or nonzero cosmological constant [42]. Since 1995 two teams of astronomers - the High-Z Supernova Search Team and the Supernova Cosmology Project - have been discovering type Ia supernovae at high redshifts. First results of both teams were published in Refs. [42] and [43].

As to a first comparison od theory with observations, we here consider the recently updated Supernovae Cosmology Project Union2 compilation [33], which is an update of the original Union compilation, now bringing together data for 719 SNeIa, drawn from 17 datasets. Of these, 557 SNeIa, spanning the redshift range $(0.015 \leq z \leq 1.55$.), pass usability cuts and outliers removal, and form the final sample used to constrain our model. We actually compare the theoretically predicted distance modulus $\mu(z)$ with the observed one, through a Bayesian approach, based on the use, as merit function, of the likelihood $\mathcal{L}=\exp \left(-\frac{1}{2} \chi^{2}\right)$. The distance modulus is defined by

$$
\mu \equiv m-M=5 \log d_{L}(z)+5 \log \left(\frac{c}{100 h}\right)+25,
$$

where $m$ is the appropriately corrected apparent magnitude including reddening, $\mathrm{K}$ correction etc., $M$ is the corresponding absolute magnitude, and $d_{L}$ is the luminosity distance in Mpc. However, in our cosmological model with variable $G$ and $\Lambda$, it is important also to include in Eq. (28) corrections describing the effect of the time variation of the gravitational constant $G$ on the luminosity of high redshift supernovae. If the local value of $G$ at the spacetime position of the most distant supernovae differs from $G_{N}$, this could in principle induce a change in the Chandrasekhar mass $M_{c h} \propto G^{-\frac{3}{2}}$. Some analytical models of the supernovae light curves predict that the peak luminosity is proportional to the mass of nickel produced during the explosion, which is a fraction of the Chandrasekhar mass. The actual fraction varies in different scenarios, but the physical mechanism of type Ia supernovae explosion always relates the energy yield to the Chadrasekhar mass. Assuming that the same mechanism for the ignition and the propagation of the burning front is valid for SNeIa at high and low redshifts, the predicted apparent magnitude will be fainter by a quantity [44]

$$
\Delta M_{G}=\frac{15}{4} \log \left(\frac{G}{G_{0}}\right) .
$$

Taking this into account the distance modulus becomes

$$
m-M=5 \log d_{L}(z)+5 \log \left(\frac{c}{100 h}\right)+25+\Delta M_{G} .
$$


The presence of this correction allows us to appropriately test our model by using the SNeIa sample [44] [45]] [46].

In our flat and homogeneous cosmological model the luminosity distance can be expressed as an integral of the Hubble function as follows:

$$
d_{L}(z)=\frac{c}{H_{0}}(1+z) \int_{0}^{z} \frac{1}{H(\zeta)} d \zeta
$$

where $H(z)$ is the Hubble function expressed in terms of redshift. It turns out that the luminosity distance can be expressed as a function of time in the following way:

$$
\begin{aligned}
& d_{L}(t)=-\frac{6(B+2) n^{2}(t-1)(B+1)^{2 n-1}\left(t^{\frac{6 n}{6 n-1}}\left(B+t^{\frac{6 n}{6 n-1}}\right)\right)^{-n}}{6 n-1} \\
& \times\left(\frac { 1 } { 6 n ^ { 2 } - 6 n + 1 } \left(( - 1 + 6 n ) \left(\left(\frac{1}{B}+1\right)^{n}(B+1)^{-n}{ }_{2} F_{1}\left[-n+1-\frac{1}{6 n}, n ;-n+2-\frac{1}{6 n} ;-\frac{1}{B}\right]\right.\right.\right. \\
& \left.\left.\left.\times\left(\frac{B+t^{\frac{6 n}{6 n-1}}}{B}\right)^{n}\left(t^{\frac{6 n}{6 n-1}}\left(B+t^{\frac{6 n}{6 n-1}}\right)\right)^{-n}{ }_{2} F_{1}\left[-n+1-\frac{1}{6 n}, n ;-n+2-\frac{1}{6 n} ;-\frac{t^{\frac{6 n}{6 n-1}}}{B}\right]\right)\right)\right)
\end{aligned}
$$

where ${ }_{2} F_{1}$ is an hypergeometric function. On inverting the relation $z(t)$ in Eq. (17), we obtain

$$
\begin{aligned}
& t(z)=2^{\frac{1}{6 n}-1}\left(\left((z+1)(B+1)^{-n}\right)^{-1 / n}\right. \\
& \left.\times \sqrt{\left((z+1)(B+1)^{-n}\right)^{\frac{1}{n}}\left(B^{2}\left((z+1)(B+1)^{-n}\right)^{\frac{1}{n}}+4\right)}-B\right)^{1-\frac{1}{6 n}},
\end{aligned}
$$

and we can construct $d_{L}(z)$ and evaluate the distance modulus according to Eq. (30), so as to perform our likelihood analysis, by maximizing the likelihood $\mathcal{L}=\exp \left(-\frac{1}{2} \chi^{2}\right)$ on a grid in the space of parameters [58] $B$ and $n$. In order to constrain the parameters of our model only, when we perform our statistical analysis with the Union2 compilation, we marginalize over $h$, that is, we maximize the likelihood $\mathcal{L}_{\text {marg }}=\int_{h_{\min }}^{h_{\max }} d h \exp \left(-\frac{1}{2} \chi^{2}\right)$, where $h_{\min }$ and $h_{\text {max }}$ are fixed by using the latest WMAP7 results. We obtain $\chi_{\text {reduced }}^{2}=0.97$ for 557 datapoints and the regions of confidence at $3 \sigma$ for $H_{0}$ and $\Omega_{m}$ are $(0.92,1.01)$ and $(0.24,0.4)$, respectively. If we do not marginalize over $\mathrm{h}$, we obtain $h_{b e s t}=0.70_{-0.02}^{+0.02}$, from which we can infer the following interval of confidence at $3 \sigma: \tau \in(12.8,14.7)$ Gyr. In Fig. 3 we plot the Union2 data set with the best fit modulus of distance, showing that they are, indeed, well-fitted by our model. 


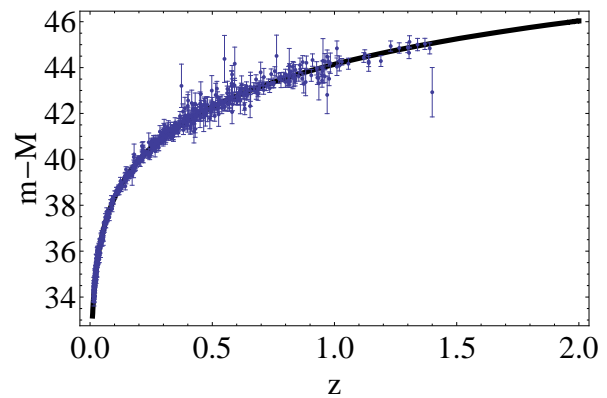

FIG. 3: The fit of the Union2 data set with the theoretical modulus of distance $\mu$ with respect to redshift $z$.

\section{CONSTRAINTS FROM CALIBRATED GAMMA RAY BURSTS HUBBLE DIA- GRAM}

Let us now assess another set of observations which we think essential to begin to understand how much our theoretical model can be considered as a reliable one. As a matter of fact, it has been recently empirically established that some of the directly observed parameters of Gamma Ray Bursts are connected with the isotropic absolute luminosity $L_{i s o}$, the collimation corrected energy $E_{\gamma}$, or the isotropic bolometric energy $E_{\text {iso }}$ of a GRB. Such observable properties of the GRBs include the peak energy, denoted by $E_{\mathrm{p}, \mathrm{i}}$, which is the photon energy at which the $\nu F_{\nu}$ spectrum is brightest; the jet opening angle, denoted by $\theta_{\text {jet}}$, which is the rest-frame time of the achromatic break in the light curve of an afterglow; the time lag, denoted by $\tau_{l a g}$, which measures the time offset between high and low energy GRB photons arriving on Earth; and the variability, denoted by $V$, which is the measurement of the spikiness or smoothness of the GRB light curve. In the literature, there is a wide variety of choices for the definition of $V$ [47] in which the observed $V$ value varies as the inverse of the time stretching, so the corresponding measured value should be multiplied by a correcting factor $(1+z)$. An additional luminosity indicator is the minimum rise time [47] denoted by $\tau_{r t}$, and taken to be the shortest time over which the light curve rises by half the peak flux of the pulse.

These quantities appear to correlate with the GRB isotropic luminosity, its total 
collimation-corrected or its isotropic energy. This property cannot be measured directly but rather it can be obtained through the knowledge of either the bolo-metric peak flux, denoted by $P_{b o l o}$, or the bolo-metric fluence, denoted by $S_{b o l o}$. Therefore, the isotropic luminosity is given by

$$
L_{\text {iso }}=4 \pi d_{L}^{2}(z) P_{\text {bolo }},
$$

the total isotropic energy reads as

$$
E_{\text {iso }}=4 \pi d_{L}^{2}(z) S_{\text {bolo }}(1+z)^{-1}
$$

and the total collimation-corrected energy is

$$
E_{\gamma}=4 \pi d_{L}^{2}(z) S_{\text {bolo }} F_{\text {beam }}(1+z)^{-1}
$$

where $F_{\text {beam }}$ is the beaming factor. The correlation relations are power-law relations of either $L_{\text {iso }}$ or $E_{\gamma}$ or $E_{\text {iso }}$ as a function of $\tau_{\text {lag }}, V, E_{\text {peak }}, \tau_{r t}$, i.e.

$$
\begin{aligned}
& E_{\text {iso }}=b_{\text {iso,peak }} E_{\text {peak }}^{a_{\text {isoak }}}, \\
& E_{\gamma}=b_{\gamma, \text { peak }} E_{\text {peak }}^{a_{\gamma, \text { peak }}}, \\
& L=b_{\text {peak }} E_{\text {peak }}^{a_{\text {peak }}} .
\end{aligned}
$$

Therefore, $L_{i s o}, E_{\gamma}$ and $E_{\text {iso }}$ depend not only on the GRB observables $P_{\text {bolo }}$ or $S_{\text {bolo }}$, but also on the cosmological parameters, through the luminosity distance $d_{L}(z)$. As a consequence, there is a fierce problem to overcome, since it is not immediately possible to calibrate such GRBs empirical laws, and to build up a new GRBs Hubble diagram, without assuming any a priori cosmological model (which is known as the circularity problem).

In Refs. [34] and [48] we have applied a local regression technique to estimate, in a model independent way, the distance modulus from the recently updated Union SNeIa sample, containing 557 SNeIa spanning the redshift range of $0.015 \leq z \leq 1.55$. The derived calibration parameters have been used to construct an updated GRBs Hubble diagram. In particular, by using such a technique, we have fitted the so-called Amati relation (see Ref. [34] for details) and constructed an updated Gamma Ray Bursts Hubble diagram, which we call the calibrated GRBs HD, consisting of a sample of 109 objects, shown in Fig. 4. Their redshift distribution covers a broad range of $z$, from 0.033 to 8.23 , thus extending far beyond that of 


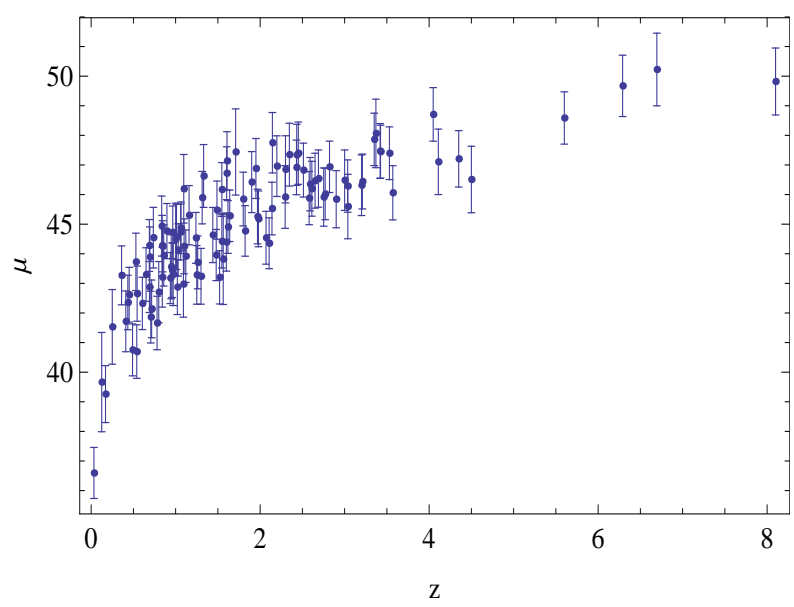

FIG. 4: Distance modulus $\mu(z)$ for the calibrated GRBs Hubble diagram made up by fitting the Amati correlation.

SNeIa $(z<\sim 1.7)$, and including GRB 092304, the new high-z record holder of Gamma Ray Bursts. Here we want to use such calibrated GRBs HD to test if our cosmological model is able to describe the background expansion up to redshifts $z \sim 8$. In our Bayesian approach to model testing, we explore the parameter space through the likelihood function

$$
\mathcal{L}_{G R B}(\mathbf{p}) \propto \exp \left[-\chi_{G R B}^{2}(\mathbf{p}) / 2\right]
$$

with

$$
\chi_{G R B}^{2}(\mathbf{p})=\sum_{i=1}^{\mathcal{N}_{G R B}}\left[\frac{\mu_{o b s}\left(z_{i}\right)-\mu_{t h}\left(z_{i}\right)}{\sqrt{\sigma_{i}^{2}+\sigma_{G R B}^{2}}}\right]^{2},
$$

where $\sigma_{G R B}$ takes into account the intrinsic scatter inherited from the scatter of GRBs around the Amati correlation (see Ref. [34] and references therein), p denotes the set of model parameters ( $B$ and $n$ and $h$ in our case), and the distance modulus $\mu(z)$ is provided by Eq. (28). The inferred confidence intervals $($ at $3 \sigma)$ for $H_{0}, \Omega_{m}$ and $h$, are $H_{0} \in(0.96,1.1)$, $\Omega_{m} \in(0.26,0.39)$, and $h \in(0.65,0.74)$. We obtain $\chi_{\text {red }}^{2}=0.97$ for 109 data points. In Fig. 5 we show the GRBs Hubble diagram with overplotted the distance modulus predicted by the fiducial model. It turns out that our cosmological model is fully compatible with this recently compiled GRBs HD. 


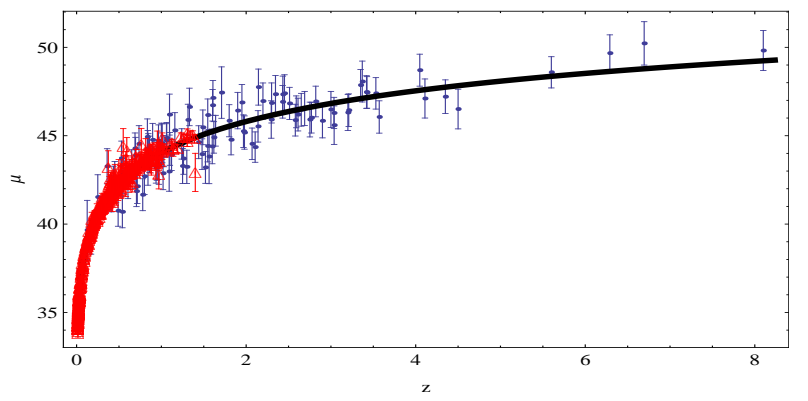

FIG. 5: The calibrated GRBs Hubble diagram with overplotted the distance modulus predicted by the fiducial model (solid line). The full circles correspond to the GRBS data set, while the empty red triangles correspond to the Union2 SneIa data points.

\section{CONSTRAINTS FROM CHANDRA X-RAY OBSERVATIONS OF LARGE RE- LAXED GALAXY CLUSTERS}

The matter content of the largest clusters of galaxies is expected to provide an almost fair sample of the matter content of the Universe (see, for instance, Refs. [49] and [35]). The ratio of baryonic-to-total mass in clusters should, therefore, closely match the ratio of the cosmological parameters $\Omega_{\mathrm{b}} / \Omega_{\mathrm{m}}$. The baryonic mass content of clusters is dominated by the X-ray emitting gas, the mass of which exceeds the mass of optically luminous material by a factor $\sim 6$, with other sources of baryonic matter being negligible. The combination of robust measurements of the baryonic mass fraction in clusters from X-ray observations together with a determination of $\Omega_{\mathrm{b}}$ from other measuremets (as for instance cosmic microwave background (CMB) data or big-bang nucleosynthesis calculations) and a constraint on the Hubble constant, can therefore be used to measure $\Omega_{\mathrm{m}}$ and constrain the parameters which characterize any cosmological model. This constraint originates from the dependence of the $f_{\text {gas }}$ measurements, which derive from the observed X-ray gas temperature and density profiles, on the assumed distances to the clusters, $f_{\text {gas }} \propto d^{1.5}$.

To understand the origin of the $f_{\text {gas }} \propto d^{1.5}$ dependence, consider a spherical region of observed angular radius $\theta$, within which the mean gas mass fraction is measured. The physical size, $R$, is related to the angle $\theta$ as $R=\theta d_{\mathrm{A}}$. The X-ray luminosity emitted from 
within this region, $L_{\mathrm{X}}$, is related to the detected flux, $F_{\mathrm{X}}$, as $L_{\mathrm{X}}=4 \pi d_{\mathrm{L}}^{2} F_{\mathrm{X}}$, where $d_{\mathrm{L}}$ is the luminosity distance and $d_{\mathrm{A}}=d_{\mathrm{L}} /(1+z)^{2}$ is the angular diameter distance. Since the $\mathrm{X}$-ray emission is primarily due to collisional processes (bremsstrahlung and line emission) and is optically thin, we may also write $L_{\mathrm{X}} \propto n^{2} V$, where $n$ is the mean number density of colliding gas particles and $V$ is the volume of the emitting region, with $V=4 \pi\left(\theta d_{\mathrm{A}}\right)^{3} / 3$. On considering the cosmological distance dependences, we see that $n \propto d_{\mathrm{L}} / d_{\mathrm{A}}^{1.5}$, and that the observed gas mass within the measurement radius $M_{\text {gas }} \propto n V \propto d_{\mathrm{L}} d_{\mathrm{A}}^{1.5}$. The total mass, $M_{\text {tot }}$, determined from the X-ray data under the assumption of hydrostatic equilibrium, is such that $M_{\text {tot }} \propto d_{\mathrm{A}}$. Thus, the X-ray gas mass fraction measured within angle $\theta$ is $f_{\text {gas }}=M_{\text {gas }} / M_{\text {tot }} \propto d_{\mathrm{L}} d_{\mathrm{A}}^{0.5}$. The expectation from non-radiative hydrodynamical simulations is that for the largest $(k T \succeq 5 \mathrm{keV})$, dynamically relaxed clusters and for measurement radii beyond the innermost core $\left(r \succeq r_{2500}\right), f_{\text {gas }}$ should be approximately constant with redshift, the virial radius $r_{2500}$ being defined as the radius of a sphere such that the mean density contained within it is $\Delta=2500$ times the critical density at the halo redshift.

It is worth noting that even if the virial radius $r_{2500}$ depends on the fiducial cosmological model, in order to determine constraints on cosmological parameters it is not necessary to generate $f_{\text {gas }}$ datasets for every cosmology of interest and compare them with the expected behaviour. Indeed, it is possible to fit a single fiducial $f_{\text {gas }}$ dataset with a model that accounts for the expected apparent variation in $f_{\text {gas }}(z)$ as the underlying cosmology is varied. Let us choose the $\Lambda \mathrm{CDM}$ reference cosmology. Following Ref. [35], the model fitted to the reference $\Lambda \mathrm{CDM}$ data is

$$
f_{\mathrm{gas}}^{\Lambda \mathrm{CDM}}(z)=\frac{K \mathcal{A} \gamma b(z)}{1+s(z)}\left(\frac{\Omega_{\mathrm{b}}}{\Omega_{\mathrm{m}}}\right)\left[\frac{d_{\mathrm{A}}^{\Lambda \mathrm{CDM}}(z)}{d_{\mathrm{A}}(z)}\right]^{1.5},
$$

where $d_{\mathrm{A}}(z)$ and $d_{\mathrm{A}}^{\Lambda \mathrm{CDM}}(z)$ are the angular diameter distances to the clusters in our test and reference cosmological models, respectively.

In order to construct the angular diameter distance for our cosmological model, we use the relation between the angular diameter distance $d_{\mathrm{A}}$ and the luminosity distance $d_{L}$

$$
d_{\mathrm{L}}=(1+z)^{2} d_{\mathrm{A}}
$$

$d_{\mathrm{L}}$ being given in Eq. (33). In Eq. (40) $\mathcal{A}$ takes into account the change in angle subtended by $r_{2500}$ as the underlying cosmology is varied, and can be evaluated as

$$
\mathcal{A}=\left(\frac{\theta_{2500}^{\Lambda \mathrm{CDM}}}{\theta_{2500}}\right)^{\eta} \approx\left(\frac{H(z) d_{\mathrm{A}}(z)}{\left[H(z) d_{\mathrm{A}}(z)\right]^{\Lambda \mathrm{CDM}}}\right)^{\eta} .
$$




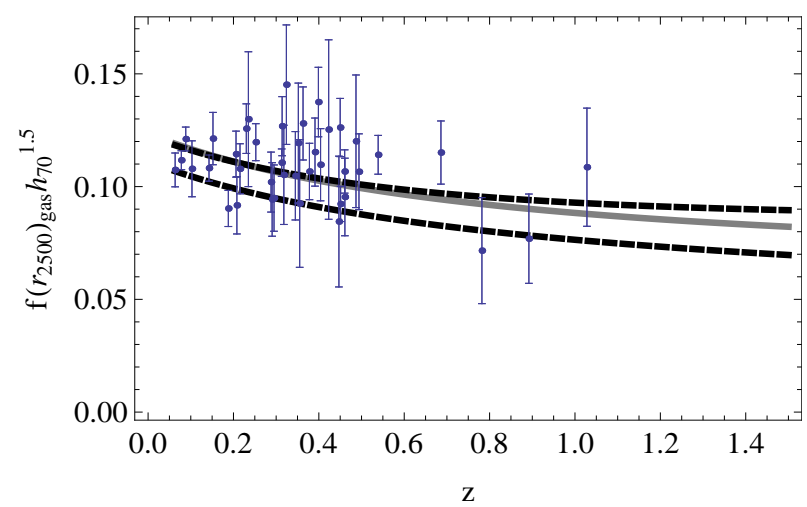

FIG. 6: The variation of the X-ray gas mass fraction measured within $r_{2500}$ as a function of redshift for our model. The dashed lines, which outline the maximum likelihood region, correspond to the extreme values for the parameters $\alpha_{\mathrm{s}}$ and $\alpha_{\mathrm{b}}$, while the gray solid line corresponds to $\alpha_{\mathrm{s}}=\alpha_{\mathrm{b}}=0$.

We take the value of the slope, $\eta$, of the $f g a s\left(r / r_{2500}\right)$ data in the region of $r_{2500}$ (as measured for the reference $\Lambda \mathrm{CDM}$ cosmology), indicated in Ref. [35], that is $\eta=0.214 \pm 0.022$. The parameter $\gamma$ in Eq. (40) models non-thermal pressure support in the clusters. On relying upon hydrodynamical simulations, we take $1.0<\gamma<1.2$. The parameter $s(z)=s_{0}\left(1+\alpha_{\mathrm{s}} z\right)$ in Eq. (400) models instead the contribution to the baryonic mass given by stars. The factor $b(z)=b_{0}\left(1+\alpha_{\mathrm{b}} z\right)$ is the 'depletion' or 'bias' factor and describes, in a completely empirical way, the ratio by which the baryon fraction measured at $r_{2500}$ is depleted with respect to the universal mean. According to Ref. [35] we choose $s_{0}=(0.16 \pm 0.05) h_{70}^{0.5},-0.2<\alpha_{\mathrm{s}}<0.2$, $0.65<b_{0}<1.0$ and $-0.1<\alpha_{b}<0.1$ (which corresponds to a moderate, systematic evolution in $b(z))$. The factor $K$ in Eq. (401) is a calibration constant fixed to $K=1.0 \pm 0.1$.

As above, we perform a Bayesian analysis, maximizing our likelihood $\mathcal{L}=\exp \left(-\frac{1}{2} \chi^{2}\right)$ on a grid in the space of parameters $B$ and $n$, and varying all the astrophysical parameters appearing in Eq. (40). Moreover, as in Ref. [35], we use the standard priors with $\Omega_{\mathrm{b}} h^{2}=$ $0.0214 \pm 0.0020$ and $h=0.72 \pm 0.08$. We obtain, in such a way, a sort of maximum likelihood region, where $f\left(r_{2500}\right)_{\text {gas }} h^{1.5}$ can vary, as shown in Fig. 6. The inferred region of confidence (at $3 \sigma$ ) for $H_{0}$ and $\Omega_{\mathrm{m}}$ are $(0.85,1.05)$ and $(0.26,0.51)$, respectively. 


\section{COSMOGRAPHY}

\section{General approach}

Over the last years the cosmographic approach to cosmology gained increasing interest for catching as much information as possible directly from observations, retaining the minimal priors of isotropy and spatial homogeneity and leaving aside any other assumptions. Actually, the only ingredient taken into account a priori in this approach is the FLRW line element obtained from kinematical requirements

$$
d s^{2}=-c^{2} d t^{2}+a^{2}(t)\left[\frac{d r^{2}}{1-k r^{2}}+r^{2} d \Omega^{2}\right] .
$$

By using this metric, it is possible to express the luminosity distance $d_{L}$ as a power series in the redshift parameter $z$, the coefficients of the expansion being functions of the scale factor $a(t)$ and its higher-order derivatives. Such an expansion leads to a distance-redshift relation which only relies on the assumption of the Friedmann-Lemaitre-Robertson-Walker metric, thus being fully model independent since it does not depend on the particular form of the solution of cosmic equations. For this purpose, it is convenient to introduce the following parameters:

$$
\begin{aligned}
H & =\frac{1}{a} \frac{d a}{d t} \\
q & =-\frac{1}{a H^{2}} \frac{d^{2} a}{d t^{2}} \\
j & =\frac{1}{a H^{3}} \frac{d^{3} a}{d t^{3}} \\
s & =\frac{1}{a H^{4}} \frac{d^{4} a}{d t^{4}}
\end{aligned}
$$

These parameters are usually referred to as the Hubble, deceleration, jerk [59], and snap parameters, respectively.

Their present day values (which, as usual, we will denote with a subscript 0) can be used to characterize the evolutionary status of the Universe. For example, $q_{0}<0$ denotes an accelerated expansion, while a change of sign of $j$ (in an expanding universe) signals that the acceleration starts increasing or decreasing. Most importantly, the parameters $\left\{q_{0}, j_{0}, s_{0}\right\}$ can be used to evaluate different distances in the universe. This can be achieved by inverting 
the scale factor series expansion (approximated to the fourth order in $t-t_{0}$ ) of a FLRW metric in terms of time, given in the following equation:

$$
\frac{a(t)}{a\left(t_{0}\right)}=1+H_{0}\left(t-t_{0}\right)-\frac{q_{0}}{2} H_{0}^{2}\left(t-t_{0}\right)^{2}+\frac{j_{0}}{3 !} H_{0}^{3}\left(t-t_{0}\right)^{3}+\frac{s_{0}}{4 !} H_{0}^{4}\left(t-t_{0}\right)^{4} .
$$

Therefore, one can obtain a series expansion of a distance, $D\left(t_{1}, t_{0}\right)$, travelled by a given

photon that was emitted at $t_{1}$ and detected at the current epoch $t_{0}$, in terms of the scale factor or redshift, while the coefficients of the expansion are defined through the cosmographic parameters. Such a distance can be related to several physical quantities, for example the luminosity distance, the angular diameter distance and more. These quantities can be constrained observationally through, for example, SNeIa, gravitational lenses, and possibly, GRB data. It is worth noticing, in this respect, that since the cosmographic approach is based on a Taylor expansion of the scale factor, or redshift, for data of GRB at high redshift (above $z=1$ ), it is better to use the variable $y=z /(1+z)$, introduced in Ref. [51], in such a way that $z \in(0, \infty)$ is mapped into $y \in(0,1)$, obtaining

$$
\begin{aligned}
d_{L}(y)= & \frac{c}{H_{0}}\left\{y-\frac{1}{2}\left(q_{0}-3\right) y^{2}+\frac{1}{6}\left[12-5 q_{0}+3 q_{0}^{2}-j_{0}\right] y^{3}+\frac{1}{24}\left[60-7 j_{0}\right.\right. \\
& \left.\left.-10-32 q_{0}+10 q_{0} j_{0}+6 q_{0}+21 q_{0}^{2}-15 q_{0}^{3}+s_{0}\right] y^{4}+\mathcal{O}\left(y^{5}\right)\right\} .
\end{aligned}
$$

\section{Application of cosmography to our model}

In this subsection, we will relate the parameters characterizing the model introduced in Sec. II to the cosmographic parameters $\left\{q_{0}, j_{0}, s_{0}\right\}$. By expanding our approximate luminosity distance up to the fourth order in the $y$-parameter, and comparing such an expansion with the standard expansion to the fourth order, we get the map which relates the parameters $B$ and $n$ of our model to the cosmographic parameters $q_{0}, j_{0}, s_{0}$. Actually, we find

$$
\begin{aligned}
q_{0} & =\frac{6 B(B+2) n-B(B+3)+12 n-2}{6(B+2)^{2} n^{2}}-1, \\
j_{0} & =18\left((B+2)^{3} n^{4}-54(B+2)(B(B+2)+2) n^{3}+9(B+2)(B(5 B+7)+6) n^{2}\right. \\
& \left.-3(B+1)(B(4 B+9)+8) n+(B+1)^{2}(B+2)\right) \frac{1}{18(B+2)^{3} n^{4}},
\end{aligned}
$$




$$
\begin{aligned}
s_{0} & =\left[B^{4}(2(n-3) n+1)(3(n-2) n+1)(6(n-1) n+1)+\right. \\
& +B^{3}(n(6 n(6 n(n(4 n(2 n-9)+59)-43)+85)-79)+5)+ \\
& +B^{2}(n(n(24 n(9 n(2 n(2 n-7)+19)-112)+851)-140)+9)+ \\
& +B(n(72 n(n(2 n(8(n-3) n+27)-31)+10)-115)+7)+ \\
& \left.+2(6(n-1) n+1)\left(4 n^{2}-6 n+1\right)(6 n(2 n-1)+1)\right] \frac{1}{36(B+2)^{4} n^{6}} .
\end{aligned}
$$

In order to constrain the model, we need to constrain observationally the cosmographic parameters by using appropriate distance indicators. Moreover, we must take care that the expansion of the distance related quantities in terms of $\left(q_{0}, j_{0}, s_{0}\right)$ closely follows the exact expressions over the range probed by the data used. Taking SNeIa and a fiducial $\Lambda$ CDM model as a test case, one has to check that the approximated luminosity distance deviates from the $\Lambda \mathrm{CDM}$ one by less than the measurement uncertainties up to $z \simeq 1.5$, to avoid introducing any systematic bias. Since we are interested in constraining the cosmographic parameters, we will expand the luminosity distance $D_{L}$ up to the fifth order in $z$ which indeed allows us to track the $\Lambda$ CDM expression with an error less than $1 \%$ over the full redshift range. To constrain the parameters $\left(h, q_{0}, j_{0}, s_{0}\right)$, we define the likelihood $\mathcal{L}(\mathbf{p})$ as

$$
\begin{aligned}
\mathcal{L}(\mathbf{p}) & \propto \frac{\exp \left(-\chi_{\text {SneIa } / \text { GRB }}^{2} / 2\right)}{(2 \pi)^{\frac{\mathcal{N}_{\text {SneIa } / \text { GRB }}}{2}}\left|\mathbf{C}_{\text {SneIa } / \text { GRB }}\right|^{1 / 2}} \\
& \times \frac{1}{\sqrt{2 \pi \sigma_{h}^{2}}} \exp \left[-\frac{1}{2}\left(\frac{h-h_{\text {obs }}}{\sigma_{h}}\right)^{2}\right] \\
& \times \frac{\exp \left(-\chi_{B A O}^{2} / 2\right)}{(2 \pi)^{\mathcal{N}_{B A O} / 2}\left|\mathbf{C}_{B A O}\right|^{1 / 2}} \\
& \times \frac{1}{\sqrt{2 \pi \sigma_{\mathcal{R}}^{2}}} \exp \left[-\frac{1}{2}\left(\frac{\mathcal{R}-\mathcal{R}_{o b s}}{\sigma_{\mathcal{R}}}\right)^{2}\right] \\
& \times \frac{\exp \left(-\chi_{H}^{2} / 2\right)}{(2 \pi)^{\mathcal{N}_{H} / 2}\left|\mathbf{C}_{H}\right|^{1 / 2}},
\end{aligned}
$$

where

$$
\chi_{\text {SneIa } / G R B}^{2}(\mathbf{p})=\sum_{i=1}^{\mathcal{N}_{\text {SneIa } / G R B}}\left[\frac{\mu_{\text {obs }}\left(z_{i}\right)-\mu_{t h}\left(z_{i}, \mathbf{p}\right)}{\sigma_{i}}\right]^{2}
$$

As observational dataset we actually use the currently available observational SNIa and GRB Hubble Diagrams, and we set Gaussian priors on the distance from Baryon Acoustic 
Oscillations (BAO), and the Hubble constant $h$. Such priors have been included in order to help break the degeneracies among the parameters of the cosmographic series expansion. In Eq. (54) $\mathbf{C}_{\text {SneIa/GRB }}$ is the SneIa/GRBs diagonal covariance matrix and $\left(h_{o b s}, \sigma_{h}\right)=$ $(0.742,0.036)$. The third term takes into account the constraints on $d_{z}=r_{s}\left(z_{d}\right) / D_{V}(z)$ with $r_{s}\left(z_{d}\right)$ the comoving sound horizon at the drag redshift $z_{d}$ (which we fix to be $r_{s}\left(z_{d}\right)=$ 152.6 Mpc from WMAP7) and the volume distance is defined as in Ref. [52]:

$$
D_{V}(z)=\left\{\frac{c z}{H(z)}\left[\frac{D_{L}(z)}{1+z}\right]^{2}\right\}^{1 / 3} .
$$

The values of $d_{z}$ at $z=0.20$ and $z=0.35$ have been estimated in Ref. [53] using the SDSS DR7 galaxy sample so that we define $\chi_{B A O}^{2}=\mathbf{D}^{T} \mathbf{C}_{B A O}^{-1} \mathbf{C}$ with $\mathbf{D}^{T}=\left(d_{0.2}^{o b s}-d_{0.2}^{t h}, d_{0.35}^{\text {obs }}-d_{035}^{t h}\right)$ and $\mathbf{C}_{B A O}$ the BAO covariance matrix. The next term refers to the shift parameter [54] [55]:

$$
\mathcal{R}=\sqrt{\Omega_{M}} \int_{0}^{z_{\star}} \frac{d z^{\prime}}{E\left(z^{\prime}\right)}
$$

with $z_{\star}=1090.10$ the redshift of the last scattering surface. We follow again WMAP7 by setting $\left(\mathcal{R}_{o b s}, \sigma_{\mathcal{R}}\right)=(1.725,0.019)$. Thus we obtain the following confidence region (at $\left.3 \sigma\right)$ for the cosmographic parameters: $\left(\begin{array}{ccc}q_{0} & -0.76 & -0.25 \\ j_{0} & 0.28 & 0.33 \\ s_{0} & -2.15 & -1.18\end{array}\right)$ confirming an accelerated stage of the universe.

In addition, we can investigate the possibility to use high redshift GRBs to determine parameters of our cosmography. As a matter of fact, we use a whole dataset containing both the SNIa Union2 dataset and the calibrated GRBs HD, which we call the cosmographic dataset. We obtain the following confidence region (at $3 \sigma$ ) for the cosmographic parameters: $\left(\begin{array}{ccc}q_{0} & -0.6 & -0.2 \\ j_{0} & 0.01 & 0.21 \\ s_{0} & -2.14 & 0.86\end{array}\right)$. In Fig. 7we actually show the cosmographic distance modulus together with the cosmographic dataset.

As a final step, we can use the re-parametrization of our model in terms of $H_{0}$ and $\Omega_{m}$ exhibited in the subsection (a) to construct $d_{L}^{\text {cosmographic }}\left(y, H_{0}, \Omega_{m}, h\right)$, and perform the cosmographic analysis using both Supernovae and Gamma Ray Bursts data (the so-called cosmographic dataset), which allow us to obtain constraints (even if not yet stringent) on the parameters of cosmography. Actually, it turns out that

$$
H_{0}=0.98_{-0.05}^{+0.04}, \quad \Omega_{m}=0.2 \pm 0.05,
$$




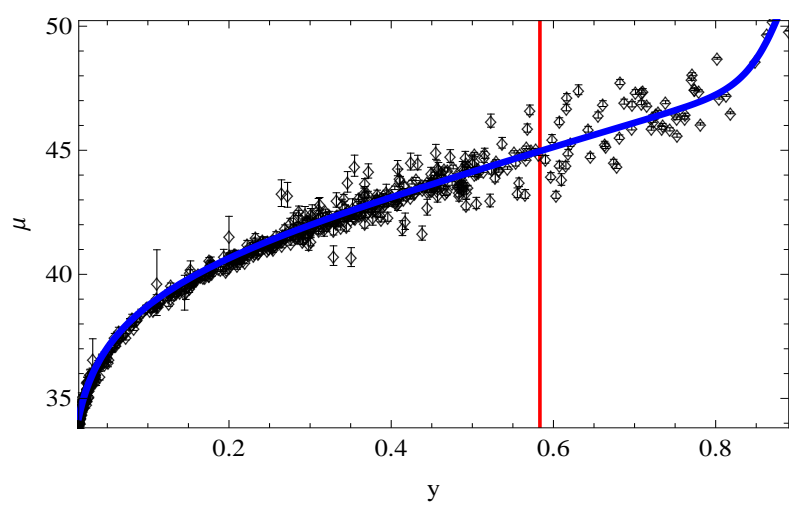

FIG. 7: Distance modulus for the best-fit values of our cosmography (solid blue line) performed with the cosmographic dataset (empty black diamond). The red vertical line indicates the extreme value of the parameter $y=z /(1+z)$ where we have SNeIa data.

and then, from Eqs. (24), we obtain the following confidence region (at $3 \sigma$ ) for the cosmographic parameters: $\left(\begin{array}{ccc}q_{0} & -0.77 & -0.26 \\ j_{0} & 0.11 & 0.39 \\ s_{0} & -2.2 & -0.64\end{array}\right)$. In Fig 7 we plot the distance modulus for the best-fit values of our cosmography (solid blue line) performed with the cosmographic dataset (empty black diamond). The red vertical line indicates the extreme value of the parameter $y=z /(1+z)$ where we have SNeIa data.

\section{A MORE REALISTIC DESCRIPTION}

The model of the universe adopted so far is described by an exact solution of the dynamical equations, whose arbitrary parameters are determined by specifying the initial conditions. In this section we now consider more realistically the inclusion of radiation, too, into such a model. In this case the dynamical equations, as far as we know, do not have analytical solutions, and therefore we will rely on numerical computations.

The dynamical field equations become

$$
\begin{gathered}
\frac{\ddot{a}}{a}+\frac{\dot{a}^{2}}{2 a^{2}}-\frac{\Lambda}{2}-\frac{\dot{a} \dot{G}}{a G}+\frac{\mu \dot{G}^{2}}{4 G^{2}}+4 \pi G\left(\gamma_{r a d}-1\right) D_{r a d} a^{-3 \gamma_{r a d}}=0, \\
\mu \ddot{G}-\frac{3}{2} \mu \frac{\dot{G}^{2}}{G}+3 \mu \frac{\dot{a}}{a} \dot{G}+\frac{G}{2}\left(-6 \frac{\dot{a}^{2}}{a^{2}}-2 \Lambda+2 G \frac{d \Lambda}{d G}\right)=0 .
\end{gathered}
$$


We have also to consider the Hamiltonian constraint [27]

$$
\frac{\dot{a}^{2}}{a^{2}}-\frac{\Lambda}{3}-\frac{\mu}{6} \frac{\dot{G}^{2}}{G^{2}}-\frac{8 \pi G}{3}\left(D_{m} a^{-3 \gamma_{m}}+D_{r a d} a^{-3 \gamma_{r a d}}\right)=0 .
$$

It turns out that these equations assume a simpler form when, instead of $t$ as an independent variable, one uses $a(t)$ - the scale factor. We introduce a new independent variable by $u \equiv \log (1+z)=-\log \left(\frac{a(t)}{a_{0}}\right)$, where $a_{0}$ is the present value of the scale factor (fixed at $\left.a_{0}=1\right)$ and $z$ is the redshift. The equations can now be written in the form

$$
\begin{gathered}
H^{2}(u)\left(1-\frac{2}{3} \mu \frac{H^{\prime}(u)}{H(u)}\right)=\frac{\Lambda(u)}{3}+\frac{2}{3} \mu H^{2}(u)\left(\frac{G^{\prime}(u)}{G(u)}\right)^{2}+\frac{8 \pi}{3} G(u) D_{r a d} a^{-3\left(\gamma_{r a d}-1\right)}, \\
G^{\prime \prime}(u)=G^{\prime}(u)\left(3+\frac{H^{\prime}(u)}{H(u)}+\frac{3}{2} \frac{G^{\prime}(u)}{G(u)}\right)+\frac{G(u)}{\mu}\left(-3+\frac{3 n \Lambda(u)}{(1-3 n) H^{2}(u)}\right), \\
H^{2}(u)=\frac{\frac{8 \pi G(u)}{3}\left(D_{r a d} a^{-3 \gamma_{r a d}}+D_{m} a^{-3 \gamma_{m}}\right)+\frac{\Lambda}{3}}{1+\frac{\mu}{6}{\frac{G^{\prime}(u)}{G(u)}}^{2}}
\end{gathered}
$$

where $^{\prime}=\frac{d}{d u}, \gamma_{r a d}=\frac{4}{3}$, and $\gamma_{m}=1$.

Moreover, we have to remember that above, in Eq. (63), we explicitly used the dependence of $\Lambda$ on $G$, as given by applying the Noether Symmetry Approach to the case of a matterdominated universe (without any radiation content) [60]: $\Lambda(u)=W G^{\frac{1}{1-3 n}}(u)$. Let us note that Eqs. (62) and (64) can be rewritten in a Friedmann-like form

$$
\begin{gathered}
H^{2}(u)\left(1-\frac{2}{3} \frac{H^{\prime}(u)}{H(u)}\right)=-\frac{4 \pi G}{3}\left(p_{\Lambda, G}+p_{\text {rad }}\right), \\
H^{2}(u)=\frac{8 \pi G(u)}{3}\left(\rho_{\Lambda, G}+\rho_{r a d}+\rho_{m}\right),
\end{gathered}
$$

where we define

$$
\begin{gathered}
\rho_{\Lambda, G} \equiv \frac{\Lambda}{8 \pi G(u)}+\frac{H(u)^{2}\left(3-\frac{\mu}{6}\right) G^{\prime}(u)^{2}}{4 \pi G^{3}}, \\
p_{\Lambda, G} \equiv \frac{H^{2}\left(3-\frac{2 \mu}{3}\right) u^{2}\left(G^{\prime}\right)^{2}}{4 \pi G^{3}}-\frac{\Lambda}{4 \pi G},
\end{gathered}
$$

and then construct $w=\frac{p_{\Lambda, G}}{\rho_{\Lambda, G}}$. Substituting Eq. (64) in Eq. (63), we can numerically solve the system of differential equations characterizing cosmology, by specifying the initial condition at $u=70$, for example, and assuming that $G(70)$, and $G^{\prime}(70)$ have the same values as in the case without radiation. As can be seen in Fig. 8, the presence of radiation affects the evolution of the $\Omega$ parameters. As expected, at the initial time, again when $u=30$, radiation dominates the expansion rate of the universe, with the $\Lambda$-term and matter being 


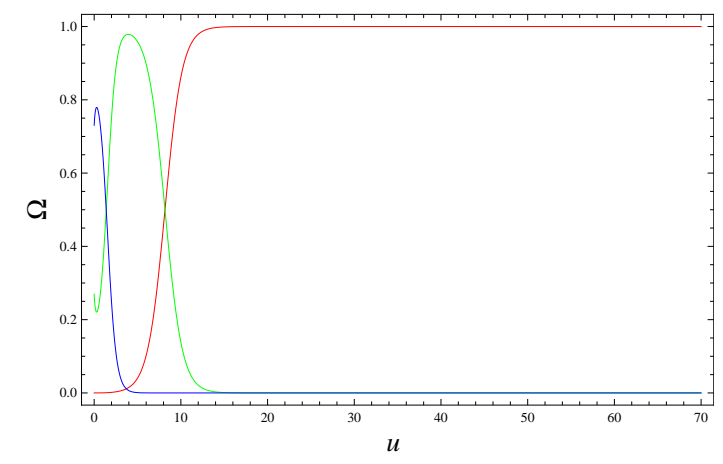

FIG. 8: $\Omega$ parameters as functions of $u$ in the universe filled in with matter, radiation and the $\Lambda$-term. $\Omega_{\Lambda}$ is marked in blue, $\Omega_{r}$ in red and $\Omega_{m}$ in green.

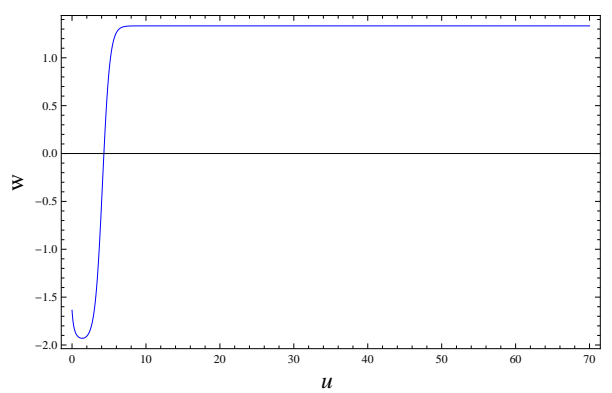

FIG. 9: The effective equation of state $w$ as a function of $u$.

subdominant, at a redshift $z$ of about 5000; the energy densities of matter and radiation become equal, and, for a relatively short period, the universe becomes matter dominated until, at a redshift of about 1 , the $\Lambda$-term starts dominating the expansion rate of the universe. In Fig. 9 we plot the behaviour of $w$ as a function of $u$ : we can therefore see that it behaves like stiff matter $\left(w=\frac{4}{3}\right)$ up to $u \simeq 10$, when there is the beginning of a transition towards a superquintessence behavior with $w<-1$.

It is worth noting that, since the initial conditions needed to numerically integrate the Eqs. (65), (66) and (67), have been set with the only constraint of obtaining a well-behaved 
evolution for the $\Omega$ parameters, the values $w(0), \Omega_{\Lambda}(0), \Omega_{r}(0)$, and $\Omega_{m}(0)$, cannot be directly compared with the ones indicated in the literature (see for instance Ref. [33]), because they do not result from a fitting procedure on observational datasets. Moreover, as far as the equation of state of dark energy is concerned, the best-fit values also depend on the mathematical law assumed for it. For instance, if we adopt models of dark energy as potential energy of some, as yet undiscovered, scalar field, we cannot obtain superquintessential values $w<-1$, unless we consider phantom fields or non-minimal coupling terms. On the other hand, such values are fully acceptable when we look at the confidence regions obtained by fitting the data with a parametrized form of the equation of state; however, this is not the same as saying that the scalar field is ruled out by the statistics.

\section{CONCLUSIONS}

We have shown that one can build a matter-dominated cosmological model with variable Newton parameter and variable cosmological term which is compatible with the more updated observations of type Ia supernovae, the gamma ray bursts Hubble diagram, and the gas mass fraction in X-ray luminous galaxy clusters. Moreover, we have applied to such a cosmological model a cosmographic approach, which can help in selecting realistic models without a priori choices that can be questionable. In performing our cosmographic analysis we set Gaussian priors on the distance from Baryon Acoustic Oscillations (BAO), and the Hubble constant $h$. Such priors have been included in order to help break the degeneracies among the parameters of the cosmographic series expansion. A more realistic approach, considering the inclusion of a radiation component, seems also possible, even if it has to be worked out only numerically.

Some questions remain, however, unsolved; for example, it is not clear enough what can be definitely said about the effects of $G$, which might point out to the violation of the strong equivalence principle, but not necessarily of the Einstein equivalence principle. Here we have simply discussed the observational bounds on the possible variations of the gravitational constant in the early universe, considering the best limit (at $3 \sigma), \delta G=0.09_{-0.19}^{+0.22}$, obtained in the literature. It turned out that our model can satisfy such a best fit limit, provided that the $n$ parameter is appropriately chosen (the role of $B$ is only marginal with respect to this strong test) to be compatible with the basic cosmological observations. We postpone 
to a forthcoming paper the detailed analysis of the the dependence of the various elemental abundances on the time variation of $G$ for our model. We expect that the BBN constraints are the main tool to restrict the allowed region of the model parameters, so representing a necessary step in the feasibility study of the model itself.

The concrete possibility to generate acceptably a previous radiation-dominated regime in the framework of Renormalization-Group inspired cosmology has still to be proved. Unfortunately, the procedure of the Noether Symmetry Approach does not work with a Lagrangian where the matter term is $L_{m} \equiv D\left(1-a^{-1}\right)$ (being $\gamma=1$ for dust and $\gamma=4 / 3$ for radiation). However, as we have seen, a numerical integration of the equations derived by using such an $L_{m}$, implies that, after inflation, such equations do give rise to a period of radiation dominance followed by one of matter dominance. Later, a period of acceleration only depending on variability of $\Lambda$ becomes the dominant one.

As far as we can see, we think that the work here presented may be considered as another important step towards a more accurate confrontation of variable- $\mathrm{G}$ cosmologies with modern observations. Even if, as said in the Introduction, such a comparison has begun earlier with many of the papers cited in the references, they all indeed only contribute to show a first feasibility of some of such cosmological models, not only from a theoretical but also from an observational point of view. Much work still remains to be done but the years to come will hopefully help in further restricting the family of these and other possibly viable cosmological models, mainly from the observational point of view. Actually, in a forthcoming paper we are going to investigate the behavior of density perturbations during the matter dominated stage.

\section{Acknowledgments}

G. Esposito and C. Rubano are grateful to the Dipartimento di Scienze Fisiche of Federico II University, Naples, for hospitality and support.

* E-mail at: ester@na.infn.it

[1] Davis, T. M., et al.: Astroph. J. 666, 716 (2007)

[2] Ostriker, J. P.: Ann. Rev. Astron. Astrophys. 31, 689 (1993) 
[3] Lusanna, L.: "Dark matter as a relativistic inertial effect in Einstein canonical gravity?" (2010) (arXiv:1011.2908 [gr-qc])

[4] Durrer,R., Maartens, R.: Gen. Relat. Grav. 40, 301 (2008)

[5] Capozziello, S., Francaviglia, M.: Gen. Relat. Grav., 40, 357 (2008)

[6] Capozziello, S., Faraoni, V.: Beyond Einstein Gravity, Springer, Dordrecht, 2011

[7] Reuter, M., Wetterich, C.: Nucl. Phys. B 427, 291 (1994)

[8] Reuter, M.: Phys. Rev. D 57, 971 (1998)

[9] Souma, W.: Prog. Theor. Phys. 102, 181 (1999)

[10] Wetterich, C.: Int. J. Mod. Phys. A 16, 1951 (2001)

[11] Berges, J., Tetradis, N., Wetterich, C.: Phys. Rep. 363, 223 (2002)

[12] Lauscher, O., Reuter, M.: Phys. Rev. D 65, 025013 (2002)

[13] Reuter, M., Saueressig, F.: Phys. Rev. D 65, 065016 (2002)

[14] Lauscher, O., Reuter, M.: Class. Quantum Grav. 19, 483 (2002)

[15] Babic, A., Guberina, B., Horvat, R., Stefancic, H.: Phys. Rev. D 71, 124041 (2005)

[16] Codello, A., Percacci, R.: Phys. Rev. D 97, 221301 (2006)

[17] Bonanno, A., Reuter, M.: JHEP 02, 035 (2005)

[18] Niedermaier, M.: Nucl. Phys. B 673, 131 (2003)

[19] Niedermaier, M.: JHEP 12, 066 (2002)

[20] Forgacs, P., Niedermaier, M.: "A fixed point for truncated quantum Einstein gravity" (2002) (hep-th/0207028)

[21] Bonanno, A., Reuter, M.: Phys. Rev. D 65, 043508 (2002)

[22] Bonanno, A., Reuter, M.: Phys. Rev. D 60, 084011 (1999)

[23] Bonanno, A., Reuter, M.: Phys. Rev. D 62, 043008 (2000)

[24] Tsamis, N. C., Woodard R. P.: Phys. Lett. B 301, 351 (2003)

[25] Bonanno, A., Reuter, M.: Phys. Lett. B 527, 9 (2002)

[26] Bentivegna, E., Bonanno, A., Reuter, M.: JCAP 01, 001 (2004)

[27] Bonanno A., Esposito, G., Rubano, C.: Class. Quantum Grav. 21, 5005 (2004)

[28] de Ritis, R., Marmo, G., Platania, G., Rubano, C., Scudellaro, P., Stornaiolo, C.: Phys. Rev. D 42, $1091(1990)$

[29] Capozziello, S., de Ritis, R., Rubano, C., Scudellaro, P.: Rivista del Nuovo Cimento 19(4), 1 (1996) 
[30] Bonanno, A., Esposito, G., Rubano, C., Scudellaro, P.: Class. Quantum Grav. 24, 1443 (2007)

[31] Bonanno, A., Esposito, G., Rubano, C., Scudellaro, P.: Int. J. Geom. Meth. Mod. Phys. 5, $329(2008)$

[32] Bonanno, A., Esposito, G., Rubano, C., Scudellaro, P.: Gen. Relat. Grav. 39, 189 (2007)

[33] Amanullah, R., et al.: Astroph. J. 716, 712 (2010)

[34] Demianski, M., Piedipalumbo, E., Rubano, C.: Mon. Not. R. Astron. Soc. 411, 1213 (2011)

[35] Allen, S. W., Rapetti, D. A., Schmidt, R. W., Ebeling, H., Morris, G., Fabian, A.C.: Mon. Not. R. Astron. Soc. 383, 879 (2008)

[36] Capozziello, S., de Ritis, R.: Gen. Relat. Grav. 29, 1425 (1997)

[37] Capozziello, S., de Ritis, R., Marino, A. A.: Gen. Relat. Grav. 30, 1247 (1998)

[38] Bonanno, A., Esposito, G., Rubano, C., Scudellaro, P.: Class. Quantum Grav. 23, 3103 (2006)

[39] Bambi, C., Giannotti, M., Villante, F. L.: Phys. Rev. D 71, 123524 (2005)

[40] Pitjeva, E. V.: Astron. Lett. 31, 340 (2005)

[41] Jarosik, N., Bennett, C. L., Dunkley, J., Gold, B., Greason, M. R.: Astroph. J. Suppl. S. 192, $14(2011)$

[42] Schmidt, B. P., et al.: Astroph. J. 507, 46 (1998)

[43] Perlmutter, S., et al.: Astroph. J. 517, 565 (1999)

[44] Gaztañaga, E., Garcia-Berro, E., Isern, J., Bravo, E., Dominguez, I.: Phys. Rev. D 65, 023506 (2002)

[45] Uzan, J. Ph.: Rev. Mod. Phys 75, 403 (2003)

[46] Rubano, C., Scudellaro, P., Piedipalumbo, E., Capozziello, S., Capone, M.: Phys. Rev. D 69, $103510(2004)$

[47] Schaefer, B. E.: Astroph. J. 660, 16 (2007)

[48] Demianski, M., Piedipalumbo, E.: "Standardizing the GRBs with the $E_{\mathrm{p}, \mathrm{i}}-E_{\text {iso }}$ relation: the updated Hubble diagram and implications for cosmography" (2011), submitted to Mon. Not. R. Astron. Soc.

[49] White, S. D. M., Navarro, J. F., Evrard, A. E., Frenk, C. S.: Nature 366, 429 (1993)

[50] Sahni, V., Saini, T. D., Starobinsky, A. A., Alam, U.: JETPL 77, 201 (2003)

[51] Chevallier, M., Polarski, D.: Int. J. Mod. Phys. D 10, 213 (2001)

[52] Eisenstein, D.J., et al.: Astroph. J. 633, 560 (2005)

[53] Percival, et al.: Mon. Not. R. Astron. Soc. 401, 2148 (2010) 
[54] Bond, J.R., Efstathiou, G., Tegmark, M.: Mon. Not. R. Astron. Soc. 291, L33 (1997)

[55] Efstathiou, G., Bond, J.R.: Mon. Not. R. Astron. Soc. 304, 75 (1999)

[56] It is worth noting that such a constraint on the parameters $p_{i}$ of our model is not strictly necessary, but it has been used, already in Ref. [31], as an instrument to fix somehow the reachable regions in the space of parameters (at least for what it concerns $n$ ). This means that one could perform our analysis leaving the $p_{i}$ parameters, and then $n$, completely free

[57] This limit refers to the value of $G$ when the temperature of the universe is $0.02 \leq T \leq 0.2$ $\mathrm{MeV}$ (i.e. during and immediately after the d-bottleneck epoch) and is consistent with the standard assumption that $G$ has lightly varied during the evolution of the universe.

[58] Let us note that in principle it could be better to use the re-parametrization described above (i.e. the dependence of $B$ and $n$ on $H_{0}$ and $\Omega_{m}$ provided by Eqs. (23) and (24)) and to maximize the likelihood with respect to the parameters $h, H_{0}$ and $\Omega_{m}$, since they have a brighter physical meaning and their space of parameters is easily reconstructed as discussed above. However, the mathematical form of $B\left(H_{0}, \Omega_{m}\right)$ and $n\left(H_{0}, \Omega_{m}\right)$, when inserted in the luminosity distance and in the correction term $\Delta M_{G}$, is such that they make the numerical calculations too hard and time consuming. Thus, we prefer to work in the space of parameters $h, B$ and $n$. Anyway, we will work in the space of parameters $h, H_{0}$ and $\Omega_{m}$ later, when we implement the cosmographic approach.

[59] The use of the jerk parameter to discriminate between different models was also proposed in the context of the statefinder parametrization [50].

[60] We actually remember that in the presence of radiation it is not possible to solve analytically the equations by defining the existence of the Noether symmetry vector field. 